Our Nature (2006)4:69-82

\title{
Use of Medicinal Plants in Traditional Tibetan Therapy System in Upper Mustang, Nepal
}

\author{
M. R. Pandey \\ National Trust for Nature Conservation-Annapurna Conservation Area Project
}

Received: 10.11.2006; Accepted: 15.12.2006

\begin{abstract}
The paper deals with 93 species of medicinal plants belonging to 74 genera spread over 35 families, which are used in traditional Tibetan therapy system by amchis (medical practitioners) in Upper Mustang.
\end{abstract}

Key words: Amchis, Medicinal plants, Tibetan therapy system, Upper Mustang

\section{Introduction}

Majority of total population of Nepal are dependent on traditional medicinal practices. According to an estimation of the World Health Organization, approximately $80 \%$ of the people in developing countries depend on traditional medicines for primary health care; a major portion of these involves the use of medicinal plants. As the traditional Himalayan medicine has affected the lives of people around the globe, Upper Mustang is also not the exception. Upper Mustang has characteristic features of Tibetan culture and environment, so Tibetan system of therapy has been practiced since time immemorial. Such type of traditional medicinal practice is a good example of traditional knowledge system, in which, parents to children passes down medicinal knowledge from generation to generation. This area has played a significant role in the historical processes of traditional Tibetan therapy system for understanding the development processes.

Upper Mustang has great wealth of medicinal plants and traditional knowledge. Medicinal value of plants is largely based on the Tibetan system of treatment and amchis are skilled persons to prescribe them. Amchis or medical practitioners are the health service providers in the remote highland districts of Nepal such as Mustang, Dolpa, Humla, Mugu, Gorkha and Rasuwa. An Amchi is very much like a doctor having multiple skills who provides diagnoses and treatments (Bista et al., 2002). Amchis are responsible for the conservation of high altitude medicinal plants having good knowledge of biology and ecology.

The study area (N $28^{\circ} 47^{\prime} 39^{\prime \prime}$ to $29^{\circ} 19^{\prime}$ $54^{\prime \prime}$ and E $83^{\circ} 28^{\prime} 55^{\prime \prime}$ to $84^{\circ} 15^{\prime} 16^{\prime \prime}$ ) is located in the Trans Himalayan region of Nepal. It is the northern part of Mustang district extended up to the Tibetan autonomous region of China to the northeast, Dolpo district to the west and by peaks reaching more than $6000 \mathrm{~m}$. to the south. It occupies the major portion (2567.55 sq. km.) of Annapurna Conservation Area (7629 sq. $\mathrm{km}$.), the largest protected area in Nepal $(33.7 \%)$. It is a cold high altitude steppe that falls in the rain shadow of Dhaulagiri Himal and the Annapurna massif to the south. The 
ethnic composition of Upper Mustang is exclusively of $L o$ (Tibetan) origin and they are called as Loba. The caste system prevails differentiating the elite and high caste Bista followed by Gurung as middle caste and then Biswokarma as occupational caste.

This study is based on the various surveys carried out by National Trust for Nature Conservation (NTNC)-Annapurna Conservation Area Project (ACAP)/ Upper Mustang Biodiversity Conservation Project (UMBCP) for the effective biodiversity conservation in Upper Mustang area.

\section{Materials and methods}

The study was carried out in all seven Village Development Committees (VDCs) - Chhoser, Chhonhup, Lo Manthang, Tsarang, Surkhang, Ghami and Chhuksang of Upper Mustang during 2004 to 2005. Interviews were conducted with various amchis of Upper Mustang, practicing both in the area and Kathmandu. The information is based on the first-hand information gathered from amchis and through personal observation of author on the use of remedies. The information was also cross-checked with different amchis. The classification of plants is based on the Flowers of Himalaya (Polunin and Stainton, 1997; Stainton, 1997).

\section{Results and discussion}

In order to provide more detailed information on the most important and therapeutically useful herbs/plants, 93 plants (Table 1) have been selected. In traditional Tibetan therapy, the herbal medicine includes mineral and animal products as well as plants, but this paper is focused only on medicinal plants that are found in Upper Mustang area. The recorded medicinal plants belong to 74 genera spread over 35 families (Table 2). Among them, Compositae is the largest family containing 9 species, followed by Labiatae (7 species). Gentianaceae, Polygonaceae and Ranunculaceae containing 6 species each and Primulaceae 5, where as Boraginaceae, Leguminosae, Rosaceae, Scrophulariaceae and Umbelliferae has 4 each.

\section{Acknowledgments}

I am indebted to amchis Ghyatsho Bista, Tenzing Bista (Lama), Rinjin Wangel Gurung, Tashi Namgyal Gurung and Tenjing Dharke Gurung and local people of Upper Mustang for their valuable help through out the study. Thanks are due to Mr. Madhu Chetri, NTNC-ACAP/UMBCP for his valuable suggestions and Ranger Mr. Basudev Neupane for collecting information.

\section{References}

Bista, T., G. Bista and Y.C. Lama. 2002. Building the capacity of the Himalayan Amchi. Improving Pastoral Livelihoods in the HKH Highlands, Vol. II, ICIMOD, pp. 131-141.

Polunin, O. and A. Stainton. 1997. Flowers of the Himalaya. ISBN 0195641876

Stainton, A.1997. Flowers of the Himalaya- A supplement. ISBN 0195644158 
M.R. Pandey / Our Nature (2006)4:69-82

\begin{tabular}{|c|c|c|c|c|c|c|c|c|}
\hline $\begin{array}{l}\mathbf{S} \\
\mathbf{N}\end{array}$ & $\begin{array}{l}\text { Plant } \\
\text { species }\end{array}$ & Family & $\begin{array}{l}\text { Tibetan/I } \\
\text { ocal name }\end{array}$ & $\begin{array}{l}\text { Nepali } \\
\text { Name }\end{array}$ & $\begin{array}{l}\text { English } \\
\text { Name }\end{array}$ & $\begin{array}{l}\text { Part } \\
\text { used }\end{array}$ & $\begin{array}{l}\text { Medicinal use } \\
\text { and action }\end{array}$ & Remarks \\
\hline 1 & $\begin{array}{l}\text { Allium } \\
\text { fasciculatum }\end{array}$ & Amaryllidaceae & $\begin{array}{l}\text { Rhi } \\
\text { Ghokpa }\end{array}$ & Limbu & Wild garlic & $\begin{array}{l}\text { Whole } \\
\text { plant }\end{array}$ & $\begin{array}{l}\text { Wounds and } \\
\text { swelling }\end{array}$ & $\begin{array}{l}\text { Used as } \\
\text { vegetable } \\
\text { and flavor }\end{array}$ \\
\hline 2 & $\begin{array}{l}\text { Allium } \\
\text { hypsistum }\end{array}$ & Amaryllidaceae & Jimnak & Jimbu & $\begin{array}{l}\text { Chives/Nep } \\
\text { al aromatic } \\
\text { leaf garlic }\end{array}$ & $\begin{array}{l}\text { Whole } \\
\text { plant }\end{array}$ & $\begin{array}{l}\text { Cough, cold, } \\
\text { gastritis and } \\
\text { tonic }\end{array}$ & $\begin{array}{l}\text { Used as } \\
\text { vegetable } \\
\text { and flavor }\end{array}$ \\
\hline 3 & $\begin{array}{l}\text { Arisaema } \\
\text { flavum }\end{array}$ & Araceae & Dahpa & Timju & $\begin{array}{l}\text { Cobra } \\
\text { plant/Cobra } \\
\text { lily }\end{array}$ & $\begin{array}{l}\text { Root, } \\
\text { rhizom } \\
\text { e and } \\
\text { flower }\end{array}$ & $\begin{array}{l}\text { Fever, stomach } \\
\text { problems, } \\
\text { swelling, skin } \\
\text { diseases, } \\
\text { scabies, bone } \\
\text { disease, uterus } \\
\text { and menstrual } \\
\text { disorders }\end{array}$ & $\begin{array}{l}\text { Used as } \\
\text { vegetable }\end{array}$ \\
\hline 4 & $\begin{array}{l}\text { Arisaema } \\
\text { jacquemontii }\end{array}$ & Araceae & Dahpa & Timju & $\begin{array}{l}\text { Cobra } \\
\text { plant/Cobra } \\
\text { lily }\end{array}$ & $\begin{array}{l}\text { Root, } \\
\text { rhizom } \\
\text { e and } \\
\text { flower }\end{array}$ & $\begin{array}{l}\text { Fever, stomach } \\
\text { problems, } \\
\text { swelling, } \\
\text { toothache, } \\
\text { scabies, chest } \\
\text { infection, } \\
\text { uterus and } \\
\text { menstrual } \\
\text { disorders, } \\
\text { anthelmintic } \\
\text { and throat } \\
\text { problems }\end{array}$ & $\begin{array}{l}\text { Used as } \\
\text { vegetable }\end{array}$ \\
\hline 5 & $\begin{array}{l}\text { Berberis } \\
\text { erythroclada }\end{array}$ & Berberidaceae & $\begin{array}{l}\text { Kerba } \\
\text { Nakpo }\end{array}$ & Chutro & Barberry & Bark & $\begin{array}{l}\text { Gall bladder } \\
\text { and eye } \\
\text { problems }\end{array}$ & \\
\hline 6 & Betula utilis & Betulaceae & Takpa & Bhoj Patra & $\begin{array}{l}\text { Himalayan } \\
\text { Birch }\end{array}$ & $\begin{array}{l}\text { Bark, } \\
\text { resin, } \\
\text { fruit } \\
\text { and } \\
\text { leaf }\end{array}$ & $\begin{array}{l}\text { Antiseptic, } \\
\text { hysteria, cuts, } \\
\text { wounds, burns, } \\
\text { jaundice, ear } \\
\text { pain, asthma, } \\
\text { cough, cold, } \\
\text { internal injury } \\
\text { and } \\
\text { menstruation }\end{array}$ & $\begin{array}{l}\text { Used as } \\
\text { timber } \\
\text { and fuel } \\
\text { wood }\end{array}$ \\
\hline 7 & $\begin{array}{l}\text { Incarvillea } \\
\text { arguta }\end{array}$ & Bignoniaceae & $\begin{array}{l}\text { Uag } \\
\text { Chhoy }\end{array}$ & $\begin{array}{l}\text { Surya } \\
\text { Pushpi }\end{array}$ & Bignonia & $\begin{array}{l}\text { Whole } \\
\text { plant }\end{array}$ & Tooth decay & $\begin{array}{l}\text { Plant } \\
\text { juice used }\end{array}$ \\
\hline 8 & $\begin{array}{l}\text { Incarvillea } \\
\text { younghusban } \\
\text { dii }\end{array}$ & Bignoniaceae & Ukche & $\begin{array}{l}\text { Surya } \\
\text { Pushpi }\end{array}$ & Bignonia & $\begin{array}{l}\text { Root } \\
\text { and } \\
\text { fruit }\end{array}$ & $\begin{array}{l}\text { Cough, ear } \\
\text { problem and } \\
\text { for quick } \\
\text { release of } \\
\text { placenta }\end{array}$ & \\
\hline 9 & Arnebia sp. & Boraginaceae & Mukchi & Marangi & Onosma & Root & $\begin{array}{l}\text { Fever caused } \\
\text { due to lung } \\
\text { problem, blood } \\
\text { purification, } \\
\text { hypertension, } \\
\text { cough and } \\
\text { body pain }\end{array}$ & \\
\hline
\end{tabular}


M.R. Pandey / Our Nature (2006)4:69-82

\begin{tabular}{|c|c|c|c|c|c|c|c|c|}
\hline 10 & $\begin{array}{l}\text { Cynoglossum } \\
\text { sp. }\end{array}$ & Boraginaceae & $\begin{array}{l}\text { Nema } \\
\text { Jarma }\end{array}$ & & & $\begin{array}{l}\text { Leaf, } \\
\text { stem, } \\
\text { flower } \\
\text { and } \\
\text { fruit }\end{array}$ & $\begin{array}{l}\text { Swelling, bone } \\
\text { fracture, cuts } \\
\text { and tumors }\end{array}$ & \\
\hline 11 & $\begin{array}{l}\text { Lindelofia } \\
\text { sp. }\end{array}$ & Boraginaceae & $\begin{array}{l}\text { Dewa } \\
\text { Yuchung }\end{array}$ & & & $\begin{array}{l}\text { Whole } \\
\text { plant }\end{array}$ & $\begin{array}{l}\text { Cuts, wounds } \\
\text { and swelling }\end{array}$ & \\
\hline 12 & $\begin{array}{l}\text { Maharanga } \\
\text { emodi }\end{array}$ & Boraginaceae & Dhimok & Marangi & Onosma & $\begin{array}{l}\text { Whole } \\
\text { plant }\end{array}$ & $\begin{array}{l}\text { Hypertension, } \\
\text { fever caused } \\
\text { due to lung } \\
\text { problem, blood } \\
\text { purification, } \\
\text { skin diseases, } \\
\text { rheumatism, } \\
\text { urinary } \\
\text { disorders and } \\
\text { as hair tonic }\end{array}$ & $\begin{array}{l}\text { Roots } \\
\text { mixed } \\
\text { with } \\
\text { mustard } \\
\text { oil render } \\
\text { the hair } \\
\text { black and } \\
\text { red colour } \\
\text { is } \\
\text { extracted } \\
\text { from root } \\
\text { bark and } \\
\text { is used } \\
\text { for food } \\
\text { stuff }\end{array}$ \\
\hline 13 & $\begin{array}{l}\text { Lonicera } \\
\text { hypoleuca }\end{array}$ & Caprifoliaceae & Tukser & $\begin{array}{l}\text { Masino } \\
\text { Kanike }\end{array}$ & $\begin{array}{l}\text { Honey } \\
\text { suckle/Wild } \\
\text { honey } \\
\text { suckle }\end{array}$ & $\begin{array}{l}\text { Whole } \\
\text { plant }\end{array}$ & $\begin{array}{l}\text { Kidney } \\
\text { problems }\end{array}$ & \\
\hline 14 & $\begin{array}{l}\text { Chenopodiu } \\
m \text { album }\end{array}$ & Chenopodiaceae & Niew & Bethe & $\begin{array}{l}\text { Lamb's } \\
\text { quarter/Fat } \\
\text { hen }\end{array}$ & $\begin{array}{l}\text { Seed, } \\
\text { tender } \\
\text { leaf } \\
\text { and } \\
\text { stem }\end{array}$ & $\begin{array}{l}\text { Stomach } \\
\text { trouble, piles, } \\
\text { peptic ulcer, } \\
\text { fracture, bone } \\
\text { improvement } \\
\text { and anaemia } \\
\text { fever }\end{array}$ & $\begin{array}{l}\text { Young } \\
\text { leaves are } \\
\text { eaten as } \\
\text { vegetable } \\
\text { and is a } \\
\text { good } \\
\text { source of } \\
\text { iron }\end{array}$ \\
\hline 15 & $\begin{array}{l}\text { Anaphalis } \\
\text { triplinervis }\end{array}$ & Compositae & Taygun & $\begin{array}{l}\text { Himali } \\
\text { Buki Phool }\end{array}$ & Everlasting & $\begin{array}{l}\text { Leaf } \\
\text { and } \\
\text { flower }\end{array}$ & $\begin{array}{l}\text { Food } \\
\text { poisoning, } \\
\text { diuretic and } \\
\text { heat therapy }\end{array}$ & \\
\hline 16 & $\begin{array}{l}\text { Arctium } \\
\text { lappa }\end{array}$ & Compositae & Chisyung & Tine & $\begin{array}{l}\text { Common or } \\
\text { Great } \\
\text { burdock }\end{array}$ & $\begin{array}{l}\text { Whole } \\
\text { plant } \\
\text { and } \\
\text { seed }\end{array}$ & $\begin{array}{l}\text { Intestinal } \\
\text { problem, nerve } \\
\text { building, } \\
\text { chronic } \\
\text { diseases like } \\
\text { ulcer, stomach } \\
\text { pain and } \\
\text { gastritis, fever } \\
\text { by wind, plant } \\
\text { paste is used } \\
\text { for blisters, } \\
\text { burns, pimples }\end{array}$ & \\
\hline 17 & $\begin{array}{l}\text { Artemisia } \\
\text { biennis }\end{array}$ & Compositae & $\begin{array}{l}\text { Sar Bung } \\
\text { Karpo }\end{array}$ & Tite Pati & Mug wort & $\begin{array}{l}\text { Whole } \\
\text { plant } \\
\text { root }\end{array}$ & Cough and cold & $\begin{array}{l}\text { Used as } \\
\text { incense as } \\
\text { well }\end{array}$ \\
\hline 18 & $\begin{array}{l}\text { Aster } \\
\text { stracheyi }\end{array}$ & Compositae & $\begin{array}{l}\text { Lungmi } \\
\text { Chhewa }\end{array}$ & & Aster & $\begin{array}{l}\text { Flower } \\
\text { and } \\
\text { leaf }\end{array}$ & $\begin{array}{l}\text { Wounds, } \\
\text { poisoning, } \\
\text { contagious } \\
\text { fever and } \\
\text { headache }\end{array}$ & \\
\hline
\end{tabular}


M.R. Pandey / Our Nature (2006)4:69-82

\begin{tabular}{|c|c|c|c|c|c|c|c|c|}
\hline 19 & Inula sp. & Compositae & $\begin{array}{l}\text { Mhanupat } \\
\text { a }\end{array}$ & $\begin{array}{l}\text { Puskar } \\
\text { Mul/Rasan } \\
\text { a }\end{array}$ & Inula & $\begin{array}{l}\text { Leaf } \\
\text { and } \\
\text { root }\end{array}$ & $\begin{array}{l}\text { Anaemia, } \\
\text { gastritis, fever, } \\
\text { gall bladder } \\
\text { problem, heat } \\
\text { promotion and } \\
\text { digestion }\end{array}$ & \\
\hline 20 & $\begin{array}{l}\text { Leontopodiu } \\
m \\
\text { jacotianum }\end{array}$ & Compositae & Tah & $\begin{array}{l}\text { Himali } \\
\text { Buki Phool }\end{array}$ & Edelweiss & $\begin{array}{l}\text { Flower } \\
\text { and } \\
\text { leaf }\end{array}$ & $\begin{array}{l}\text { Heat therapy } \\
\text { for cold } \\
\text { originated } \\
\text { diseases }\end{array}$ & $\begin{array}{l}\text { Used as } \\
\text { incense as } \\
\text { well }\end{array}$ \\
\hline 21 & $\begin{array}{l}\text { Saussurea } \\
\text { gossypiphora }\end{array}$ & Compositae & Kuth & $\begin{array}{l}\text { Kapase } \\
\text { Phool }\end{array}$ & $\begin{array}{l}\text { Costus/Kut } \\
\text { h }\end{array}$ & $\begin{array}{l}\text { Whole } \\
\text { plant }\end{array}$ & $\begin{array}{l}\text { Gynaecological } \\
\text { and menstrual } \\
\text { disorders, } \\
\text { hysteria, cuts, } \\
\text { bruises, cough }\end{array}$ & $\begin{array}{l}\text { Also used } \\
\text { to guard } \\
\text { against } \\
\text { evil } \\
\text { spirits }\end{array}$ \\
\hline 22 & $\begin{array}{l}\text { Soroseris } \\
\text { hookeriana }\end{array}$ & Compositae & $\begin{array}{l}\text { Solgong } \\
\text { Serpo }\end{array}$ & & & $\begin{array}{l}\text { Whole } \\
\text { plant } \\
\text { (flower } \\
\text { ) }\end{array}$ & $\begin{array}{l}\text { Fever, fracture, } \\
\text { hypertension } \\
\text { and purgative }\end{array}$ & \\
\hline 23 & $\begin{array}{l}\text { Taraxacum } \\
\text { officinale }\end{array}$ & Compositae & $\begin{array}{l}\text { Khur } \\
\text { Mang }\end{array}$ & $\begin{array}{l}\text { Tuki } \\
\text { Phool/Dud } \\
\text { he Jhar }\end{array}$ & $\begin{array}{l}\text { Bitter Wort } \\
\text { / Dandelion }\end{array}$ & Root & $\begin{array}{l}\text { Chronic } \\
\text { disorders of } \\
\text { kidney and } \\
\text { liver and also } \\
\text { used as tonic, } \\
\text { laxative and } \\
\text { antirheumatic }\end{array}$ & \\
\hline 24 & $\begin{array}{l}\text { Juniperus } \\
\text { indica }\end{array}$ & Cupressaceae & Lha shuk & Dhupi & Juniper & $\begin{array}{l}\text { Fruit } \\
\text { and } \\
\text { leaf }\end{array}$ & $\begin{array}{l}\text { Kidney, lung, } \\
\text { gall bladder } \\
\text { and liver } \\
\text { disorders, skin } \\
\text { and lymph } \\
\text { disease, fever, } \\
\text { cough, } \\
\text { paralysis of } \\
\text { limbs, disorder } \\
\text { of uterus and } \\
\text { gum swelling }\end{array}$ & $\begin{array}{l}\text { Used as } \\
\text { incense } \\
\text { and as } \\
\text { flavor in } \\
\text { some } \\
\text { cosmetics } \\
\text { and } \\
\text { drinks }\end{array}$ \\
\hline 25 & $\begin{array}{l}\text { Juniperus } \\
\text { squamata }\end{array}$ & Cupressaceae & $\begin{array}{l}\text { Syug } \\
\text { Chher }\end{array}$ & Dhupi & Juniper & $\begin{array}{l}\text { Fruit } \\
\text { and } \\
\text { leaf }\end{array}$ & $\begin{array}{l}\text { Removal of } \\
\text { accumulated } \\
\text { body fluid, } \\
\text { sleeping } \\
\text { problem, } \\
\text { kidney } \\
\text { problems, } \\
\text { bleeding, skin } \\
\text { disease and } \\
\text { wound } \\
\text { infection } \\
\end{array}$ & $\begin{array}{l}\text { Used as } \\
\text { incense as } \\
\text { well }\end{array}$ \\
\hline 26 & $\begin{array}{l}\text { Pterocephalu } \\
\text { s hookeri }\end{array}$ & Dipsacaceae & $\begin{array}{l}\text { Pangchi } \\
\text { Dhobo }\end{array}$ & $\begin{array}{l}\text { Panri } \\
\text { Mendo }\end{array}$ & & $\begin{array}{l}\text { Whole } \\
\text { plant } \\
\text { (Leaf, } \\
\text { flower } \\
\text { and } \\
\text { fruit) }\end{array}$ & $\begin{array}{l}\text { Food } \\
\text { poisoning, fluid } \\
\text { accumulation } \\
\text { in the body, } \\
\text { cough, cold, } \\
\text { contagious } \\
\text { fever, infection, } \\
\text { dysentery, } \\
\text { gout, arthritis, } \\
\text { blood disorders } \\
\end{array}$ & \\
\hline
\end{tabular}


M.R. Pandey / Our Nature (2006)4:69-82

\begin{tabular}{|c|c|c|c|c|c|c|c|c|}
\hline & & & & & & & $\begin{array}{l}\text { and intestinal } \\
\text { pain }\end{array}$ & \\
\hline 27 & $\begin{array}{l}\text { Hippophae } \\
\text { tibetana }\end{array}$ & Elaeagnaceae & Tora & $\begin{array}{l}\text { Bhuin } \\
\text { Chuk }\end{array}$ & $\begin{array}{l}\text { Seabucktho } \\
\text { rn }\end{array}$ & $\begin{array}{l}\text { Fruit } \\
\text { and } \\
\text { seed }\end{array}$ & $\begin{array}{l}\text { Gastritis, chest } \\
\text { pain, lung } \\
\text { problem, } \\
\text { digestive } \\
\text { disorder, liver } \\
\text { pain, fever, } \\
\text { blood } \\
\text { purification, } \\
\text { external use on } \\
\text { skin for bone } \\
\text { pain }\end{array}$ & $\begin{array}{l}\text { Ripe } \\
\text { fruits are } \\
\text { used to } \\
\text { make } \\
\text { juice } \\
\text { which is } \\
\text { considere } \\
\mathrm{d} \text { as } \\
\text { highly } \\
\text { nutritious }\end{array}$ \\
\hline 28 & $\begin{array}{l}\text { Ephedra } \\
\text { gerardiana }\end{array}$ & Ephedraceae & $\begin{array}{l}\text { Chhe } \\
\text { Dum }\end{array}$ & Somlata & Ephedra & $\begin{array}{l}\text { Whole } \\
\text { plant }\end{array}$ & $\begin{array}{l}\text { Hypertension, } \\
\text { cardiac trouble, } \\
\text { bleeding, } \\
\text { indigestion, } \\
\text { asthma, } \\
\text { increase blood } \\
\text { quantity in } \\
\text { body, eye } \\
\text { trouble, } \\
\text { gastritis, } \\
\text { bronchial } \\
\text { disorders, } \\
\text { cough, } \\
\text { wounds, } \\
\text { headache and } \\
\text { rheumatism }\end{array}$ & $\begin{array}{l}\text { Also an } \\
\text { important } \\
\text { source of } \\
\text { Ephedrin } \\
\text { e. This } \\
\text { plant is } \\
\text { also a } \\
\text { substitute } \\
\text { of } \\
\text { Amomum } \\
\text { subulatu } \\
m \\
\text { (Cardamo } \\
\text { m) in } \\
\text { Amchi } \\
\text { and } \\
\text { Ayurved } \\
\text { use }\end{array}$ \\
\hline 29 & $\begin{array}{l}\text { Rhododendro } \\
n \\
\text { anthopogon }\end{array}$ & Ericaceae & $\begin{array}{l}\text { Dhali } \\
\text { Karpo }\end{array}$ & Sunpati & $\begin{array}{l}\text { Rhododendr } \\
\text { on/Anthopo } \\
\text { gon leaf }\end{array}$ & $\begin{array}{l}\text { Flower } \\
\text { and } \\
\text { leaf }\end{array}$ & $\begin{array}{l}\text { Stomach, liver } \\
\text { and lung } \\
\text { disorders, T.B., } \\
\text { indigestion, } \\
\text { antiallergic } \\
\text { against potato, } \\
\text { vomiting, } \\
\text { appetizer, sore } \\
\text { throat, phlegm } \\
\text { disease, as pain } \\
\text { killer and } \\
\text { longevity }\end{array}$ & $\begin{array}{l}\text { Flowers } \\
\text { are } \\
\text { locally } \\
\text { used to } \\
\text { prepare } \\
\text { tea. } \\
\text { Essential } \\
\text { oil } \\
\text { obtained } \\
\text { from } \\
\text { leaves are } \\
\text { used in } \\
\text { high } \\
\text { grade } \\
\text { perfume. } \\
\text { Leaves } \\
\text { and } \\
\text { flowers } \\
\text { are also } \\
\text { used as } \\
\text { incense } \\
\text { with } \\
\text { juniper }\end{array}$ \\
\hline
\end{tabular}


M.R. Pandey / Our Nature (2006)4:69-82

\begin{tabular}{|c|c|c|c|c|c|c|c|c|}
\hline 30 & $\begin{array}{l}\text { Rhododendro } \\
n \text { lepidotum }\end{array}$ & Ericaceae & $\begin{array}{l}\text { Dhali } \\
\text { Karpo }\end{array}$ & $\begin{array}{l}\text { Sano } \\
\text { Chimal }\end{array}$ & $\begin{array}{l}\text { Rhododendr } \\
\text { on }\end{array}$ & $\begin{array}{l}\text { Flower } \\
\text { and } \\
\text { leaf }\end{array}$ & $\begin{array}{l}\text { Digestion, } \\
\text { appetizer, bile } \\
\text { and lung } \\
\text { diseases, back } \\
\text { pain, cold and } \\
\text { blood disorders } \\
\text { and bone } \\
\text { diseases }\end{array}$ & $\begin{array}{l}\text { Leaves } \\
\text { are used } \\
\text { as incense }\end{array}$ \\
\hline 31 & $\begin{array}{l}\text { Rhododendro } \\
n \text { nivale }\end{array}$ & Ericaceae & $\begin{array}{l}\text { Dhali Nag } \\
\text { Po }\end{array}$ & $\begin{array}{l}\text { Sano } \\
\text { Chimal }\end{array}$ & $\begin{array}{l}\text { Rhododendr } \\
\text { on }\end{array}$ & $\begin{array}{l}\text { Flower } \\
\text { and } \\
\text { leaf }\end{array}$ & $\begin{array}{l}\text { Sinusitis, } \\
\text { arthritis gout } \\
\text { and common } \\
\text { cold }\end{array}$ & \\
\hline 32 & $\begin{array}{l}\text { Gentiana } \\
\text { algida }\end{array}$ & Gentianaceae & $\begin{array}{l}\text { Pangen } \\
\text { Chhuwa }\end{array}$ & Hans Phool & Gentian & Flower & $\begin{array}{l}\text { Lung disorder } \\
\text { and skin } \\
\text { diseases }\end{array}$ & \\
\hline 33 & $\begin{array}{l}\text { Gentiana } \\
\text { robusta }\end{array}$ & Gentianaceae & $\begin{array}{l}\text { Khyi } \\
\text { Chye } \\
\text { Karpo }\end{array}$ & Hans Phool & Gentian & $\begin{array}{l}\text { Flower } \\
\text {, stem, } \\
\text { leaf } \\
\text { and } \\
\text { root }\end{array}$ & $\begin{array}{l}\text { Diarrhoea, food } \\
\text { poisoning, } \\
\text { headache, T.B., } \\
\text { urinary system } \\
\text { and bile } \\
\text { disorder, } \\
\text { swelling, cough } \\
\text { and fever }\end{array}$ & \\
\hline 34 & $\begin{array}{l}\text { Gentianella } \\
\text { moorcroftian } \\
a\end{array}$ & Gentianaceae & $\begin{array}{l}\text { Dhumpu/ } \\
\text { Tikta }\end{array}$ & & & $\begin{array}{l}\text { Whole } \\
\text { plant }\end{array}$ & Fever & $\begin{array}{l}\text { Infusion } \\
\text { of plant is } \\
\text { put on the } \\
\text { baby 's } \\
\text { forehead } \\
\text { to reduce } \\
\text { fever } \\
\text { (Febrifug } \\
\text { e) }\end{array}$ \\
\hline 35 & $\begin{array}{l}\text { Gentianella } \\
\text { paludosa }\end{array}$ & Gentianaceae & $\begin{array}{l}\text { Chyag Tig } \\
\text { Nagpo }\end{array}$ & & & Root & Headache & \\
\hline 36 & $\begin{array}{l}\text { Halenia } \\
\text { elliptica }\end{array}$ & Gentianaceae & $\begin{array}{l}\text { Tikta/Cha } \\
\text { kting }\end{array}$ & & & $\begin{array}{l}\text { Leaf } \\
\text { and } \\
\text { stem }\end{array}$ & $\begin{array}{l}\text { Liver and bile } \\
\text { disorders, } \\
\text { fever, } \\
\text { headache, } \\
\text { cough and cold }\end{array}$ & \\
\hline 37 & Swertia sp. & Gentianaceae & Tikta & Chiraito & $\begin{array}{l}\text { Swertia/Chi } \\
\text { retta }\end{array}$ & $\begin{array}{l}\text { Whole } \\
\text { plant }\end{array}$ & $\begin{array}{l}\text { Fever, } \\
\text { jaundice, } \\
\text { indigestion, } \\
\text { cough, cold, } \\
\text { blood and gall } \\
\text { bladder } \\
\text { problems }\end{array}$ & \\
\hline 38 & $\begin{array}{l}\text { Geranium } \\
\text { donianum }\end{array}$ & Geraniaceae & Ligadur & Raklamul & $\begin{array}{l}\text { Geranium/C } \\
\text { rane's bill }\end{array}$ & Root & $\begin{array}{l}\text { Fever, cough } \\
\text { and bodyache }\end{array}$ & \\
\hline 39 & $\begin{array}{l}\text { Geranium } \\
\text { nakaoanum }\end{array}$ & Geraniaceae & Ligadur & Raklamul & $\begin{array}{l}\text { Geranium/C } \\
\text { rane's bill }\end{array}$ & Root & $\begin{array}{l}\text { Fever, cough } \\
\text { and bodyache }\end{array}$ & \\
\hline 40 & $\begin{array}{l}\text { Geranium } \\
\text { pratense }\end{array}$ & Geraniaceae & $\begin{array}{l}\text { Ligadur/P } \\
\text { or }\end{array}$ & Raklamul & $\begin{array}{l}\text { Geranium/C } \\
\text { rane's bill }\end{array}$ & Root & $\begin{array}{l}\text { Fever, cough } \\
\text { and bodyache }\end{array}$ & $\begin{array}{l}\text { Not } \\
\text { considere } \\
\text { d as of } \\
\text { good } \\
\text { quality } \\
\text { compared } \\
\text { to } G \text {. }\end{array}$ \\
\hline
\end{tabular}


M.R. Pandey / Our Nature (2006)4:69-82

\begin{tabular}{|c|c|c|c|c|c|c|c|c|}
\hline & & & & & & & & $\begin{array}{l}\text { donianum } \\
\text { and } G . \\
\text { nakaoanu } \\
m\end{array}$ \\
\hline 41 & $\begin{array}{l}\text { Cordyceps } \\
\text { sinensis }\end{array}$ & Hypocreaceae & $\begin{array}{l}\text { Yarcha } \\
\text { Gumba }\end{array}$ & $\begin{array}{l}\text { Yarcha } \\
\text { gumba }\end{array}$ & & $\begin{array}{l}\text { Whole } \\
\text { plant }\end{array}$ & $\begin{array}{l}\text { Asthma, } \\
\text { aphrodisiac, } \\
\text { lung and } \\
\text { kidney } \\
\text { disorders and } \\
\text { energizer like } \\
\text { vitamin }\end{array}$ & \\
\hline 42 & $\begin{array}{l}\text { Ajuga } \\
\text { lupulina }\end{array}$ & Labiateae & Jinting & Rato Paat & Bugle & $\begin{array}{l}\text { Seed, } \\
\text { leaf } \\
\text { and } \\
\text { flower }\end{array}$ & $\begin{array}{l}\text { Fever, sinusitis, } \\
\text { infections, } \\
\text { menstrual } \\
\text { disorders, } \\
\text { swellings, skin } \\
\text { diseases and } \\
\text { paralysis }\end{array}$ & \\
\hline 43 & $\begin{array}{l}\text { Dracocephal } \\
\text { um } \\
\text { heterophyllu } \\
m\end{array}$ & Labiateae & Jibkar & Jibkar & & $\begin{array}{l}\text { Leaf } \\
\text { and } \\
\text { flower }\end{array}$ & $\begin{array}{l}\text { Oral diseases, } \\
\text { toothache and } \\
\text { liver disorder }\end{array}$ & \\
\hline 44 & $\begin{array}{l}\text { Dracocephal } \\
\text { um } \\
\text { tanguticum }\end{array}$ & Labiateae & Tiyanku & Tiyanku & & $\begin{array}{l}\text { Leaf, } \\
\text { flower } \\
\text { and } \\
\text { stem }\end{array}$ & $\begin{array}{l}\text { Fever, stomach } \\
\text { problems and } \\
\text { as blood } \\
\text { purifier }\end{array}$ & \\
\hline 45 & Elsholtzia sp. & Labiateae & $\begin{array}{l}\text { Jirung } \\
\text { Serpo }\end{array}$ & Ban Silam & & $\begin{array}{l}\text { Whole } \\
\text { plant }\end{array}$ & $\begin{array}{l}\text { Anthelmintic } \\
\text { and } \\
\text { antibacterial }\end{array}$ & \\
\hline 46 & $\begin{array}{l}\text { Phlomis } \\
\text { rotata }\end{array}$ & Labiateae & Takpak & & Phlomis & $\begin{array}{l}\text { Flower } \\
\text { and } \\
\text { leaf }\end{array}$ & $\begin{array}{l}\text { Bone problems, } \\
\text { accumulation } \\
\text { of serous fluids } \\
\text { in the bone, } \\
\text { skin and wound } \\
\text { part of the } \\
\text { body, } \\
\text { headache, } \\
\text { fever, cough, } \\
\text { worm } \\
\text { infections and } \\
\text { swelling caused } \\
\text { by cold }\end{array}$ & \\
\hline 47 & Salvia hians & Labiateae & Simthik & Aape & Sage & Leaf & Eye infection & \\
\hline 48 & $\begin{array}{l}\text { Thymus } \\
\text { linearis }\end{array}$ & Labiateae & $\begin{array}{l}\text { Maktok/M } \\
\text { akta }\end{array}$ & $\begin{array}{l}\text { Ghode } \\
\text { Marcha }\end{array}$ & Thyme & $\begin{array}{l}\text { Leaf } \\
\text { and } \\
\text { flower }\end{array}$ & $\begin{array}{l}\text { Appetizer, } \\
\text { stimulant, } \\
\text { blood purifier, } \\
\text { digestive and } \\
\text { lungs problems } \\
\text { and gum and } \\
\text { tooth problems }\end{array}$ & $\begin{array}{l}\text { Leaves } \\
\text { are used } \\
\text { as flavor } \\
\text { and } \\
\text { substitute } \\
\text { for tea } \\
\text { and chilly }\end{array}$ \\
\hline 49 & $\begin{array}{l}\text { Astragalus } \\
\text { sp. }\end{array}$ & Leguminosae & Syehekar & Bhya & & Seed & $\begin{array}{l}\text { Urine blocked, } \\
\text { intestinal pain } \\
\text { and fever }\end{array}$ & \\
\hline
\end{tabular}


M.R. Pandey / Our Nature (2006)4:69-82

\begin{tabular}{|c|c|c|c|c|c|c|c|c|}
\hline 50 & $\begin{array}{l}\text { Caragana } \\
\text { gerardiana }\end{array}$ & Leguminosae & $\begin{array}{l}\text { Jhomo } \\
\text { Shing }\end{array}$ & Kanda & Caragana & $\begin{array}{l}\text { Heart } \\
\text { wood }\end{array}$ & $\begin{array}{l}\text { Blood pressure, } \\
\text { menstrual } \\
\text { disorder, skin } \\
\text { diseases, } \\
\text { cough, heart } \\
\text { pain, blood } \\
\text { disorder and } \\
\text { eye problems }\end{array}$ & $\begin{array}{l}\text { Heart } \\
\text { wood is } \\
\text { used in } \\
\text { blood } \\
\text { pressure }\end{array}$ \\
\hline 51 & $\begin{array}{l}\text { Caragana } \\
\text { jubata }\end{array}$ & Leguminosae & $\begin{array}{l}\text { Jhomo } \\
\text { Shing }\end{array}$ & Kanda & Caragana & $\begin{array}{l}\text { Heart } \\
\text { wood }\end{array}$ & $\begin{array}{l}\text { Blood pressure } \\
\text { and menstrual } \\
\text { disorder }\end{array}$ & \\
\hline 52 & Oxytropis sp. & Leguminosae & Senak & & & $\begin{array}{l}\text { Whole } \\
\text { plant }\end{array}$ & Dog-bite & \\
\hline 53 & $\begin{array}{l}\text { Asparagus } \\
\text { sp. }\end{array}$ & Liliaceae & Nye Shing & Kurilo & Asparagus & Root & $\begin{array}{l}\text { Tonic, sexual } \\
\text { debility, } \\
\text { diarrhoea, } \\
\text { dysentery, } \\
\text { excessive } \\
\text { menstrual } \\
\text { bleeding, } \\
\text { urinogenital } \\
\text { disorders, skin } \\
\text { disease and } \\
\text { rheumatism }\end{array}$ & $\begin{array}{l}\text { Root is } \\
\text { also used } \\
\text { as an } \\
\text { alternativ } \\
\text { e to soap }\end{array}$ \\
\hline 54 & $\begin{array}{l}\text { Polygonatum } \\
\text { sp. }\end{array}$ & Liliaceae & Rahnge & Khiraunla & $\begin{array}{l}\text { Solomon's } \\
\text { Seal }\end{array}$ & Root & $\begin{array}{l}\text { Uterus } \\
\text { problem, } \\
\text { digestion, cold, } \\
\text { bile disorder, } \\
\text { aphrodisiac, } \\
\text { increase } \\
\text { regenerative } \\
\text { fluids, } \\
\text { beneficial in } \\
\text { cold wind } \\
\text { disorders and } \\
\text { as tonic to } \\
\text { restore vitality }\end{array}$ & $\begin{array}{l}\text { Roots are } \\
\text { eaten raw } \\
\text { and the } \\
\text { plant is } \\
\text { also used } \\
\text { as fixer in } \\
\text { painting } \\
\text { canvas/w } \\
\text { alls }\end{array}$ \\
\hline 55 & $\begin{array}{l}\text { Malva } \\
\text { verticillata }\end{array}$ & Malvaceae & $\begin{array}{l}\text { Chyamba } \\
\text { Pho }\end{array}$ & Lafe Saag & $\begin{array}{l}\text { Chinese } \\
\text { Mallow/Pop } \\
\text { py mallow }\end{array}$ & $\begin{array}{l}\text { Stem, } \\
\text { leaf, } \\
\text { fruit } \\
\text { and } \\
\text { flower }\end{array}$ & $\begin{array}{l}\text { Removal for } \\
\text { accumulated } \\
\text { body fluid and } \\
\text { treatment for } \\
\text { wound } \\
\text { infection, fever, } \\
\text { diarrhoea, } \\
\text { kidney and } \\
\text { urinary } \\
\text { problems }\end{array}$ & $\begin{array}{l}\text { Also used } \\
\text { as } \\
\text { vegetable }\end{array}$ \\
\hline 56 & $\begin{array}{l}\text { Dactylorhiza } \\
\text { hatagirea }\end{array}$ & Orchidaceae & Wang-lak & $\begin{array}{l}\text { Panch } \\
\text { Aunle }\end{array}$ & Orchid & $\begin{array}{l}\text { Rhizo } \\
\text { me }\end{array}$ & $\begin{array}{l}\text { Dysentery, } \\
\text { dirrhoea, skin } \\
\text { burn, chronic } \\
\text { fever, eye pain, } \\
\text { expectorant, } \\
\text { aphrodisiac and } \\
\text { nervine tonic }\end{array}$ & \\
\hline 57 & $\begin{array}{l}\text { Corydalis } \\
\text { megacalyx }\end{array}$ & Papaveraceae & $\begin{array}{l}\text { Tonzil } \\
\text { Serpo }\end{array}$ & $\begin{array}{l}\text { Bhoot } \\
\text { Kesh/Karm } \\
\text { e Jhar }\end{array}$ & Corydalis & $\begin{array}{l}\text { Whole } \\
\text { plant }\end{array}$ & $\begin{array}{l}\text { Chronic fever, } \\
\text { jaundice, bile } \\
\text { fever, wounds, } \\
\text { cough, ulcer }\end{array}$ & \\
\hline
\end{tabular}


M.R. Pandey / Our Nature (2006)4:69-82

\begin{tabular}{|c|c|c|c|c|c|c|c|c|}
\hline & & & & & & & $\begin{array}{l}\text { and blood } \\
\text { disorder }\end{array}$ & \\
\hline 58 & $\begin{array}{l}\text { Meconopsis } \\
\text { horridula }\end{array}$ & Papaveraceae & $\begin{array}{l}\text { Aachyak/ } \\
\text { Serngon }\end{array}$ & Kyaser & $\begin{array}{l}\text { Himalayan } \\
\text { Blue Poppy }\end{array}$ & $\begin{array}{l}\text { Root, } \\
\text { leaf } \\
\text { and } \\
\text { flower }\end{array}$ & $\begin{array}{l}\text { Bone fracture, } \\
\text { kidney } \\
\text { problems, } \\
\text { remove } \\
\text { accumulated } \\
\text { body fluid and } \\
\text { as pain killer }\end{array}$ & \\
\hline 59 & $\begin{array}{l}\text { Plantago } \\
\text { major }\end{array}$ & Plantagiaceae & Tharam & Isabgol & Ripple grass & $\begin{array}{l}\text { Seed, } \\
\text { leaf } \\
\text { and } \\
\text { root }\end{array}$ & $\begin{array}{l}\text { Diarrhoea, } \\
\text { dysentery, } \\
\text { intestinal } \\
\text { problems, } \\
\text { fever, bruises } \\
\text { and as tonic }\end{array}$ & \\
\hline 60 & Bistorta sp. & Polygonaceae & $\begin{array}{l}\text { Lakang/Pa } \\
\text { ngram }\end{array}$ & $\begin{array}{l}\text { Khaldi/Rak } \\
\text { tyaulo }\end{array}$ & $\begin{array}{l}\text { Hypertensio } \\
\mathrm{n}, \\
\text { diarrhoea, } \\
\text { lung and } \\
\text { intestinal } \\
\text { disorder }\end{array}$ & $\begin{array}{l}\text { Root } \\
\text { and } \\
\text { fruit }\end{array}$ & $\begin{array}{l}\text { Blood pressure, } \\
\text { diarrhoea, lung } \\
\text { and intestinal } \\
\text { disorders }\end{array}$ & \\
\hline 61 & $\begin{array}{l}\text { Oxyria } \\
\text { digyna }\end{array}$ & Polygonaceae & $\begin{array}{l}\text { Chhumach } \\
\mathrm{i}\end{array}$ & $\begin{array}{l}\text { Sano } \\
\text { Padamchal }\end{array}$ & $\begin{array}{l}\text { Mountain } \\
\text { sorrel }\end{array}$ & $\begin{array}{l}\text { Rhizo } \\
\text { me, } \\
\text { leaf } \\
\text { and } \\
\text { flower }\end{array}$ & $\begin{array}{l}\text { Fracture, joint } \\
\text { pain, lymph } \\
\text { disorder and } \\
\text { urine retention }\end{array}$ & \\
\hline 62 & $\begin{array}{l}\text { Rheum } \\
\text { australe }\end{array}$ & Polygonaceae & Chutra & Padamchal & $\begin{array}{l}\text { Himalayan } \\
\text { rhubarb }\end{array}$ & $\begin{array}{l}\text { Whole } \\
\text { plant } \\
\text { (Root } \\
\text { and } \\
\text { petiole } \\
\text { ) }\end{array}$ & $\begin{array}{l}\text { Bone fractures, } \\
\text { sprain, } \\
\text { indigestion, } \\
\text { bloated } \\
\text { stomach, } \\
\text { gastritis, } \\
\text { swellings, } \\
\text { sores, } \\
\text { menstrual and } \\
\text { blood } \\
\text { disorders, bile } \\
\text { fever and as } \\
\text { anthelmintic }\end{array}$ & $\begin{array}{l}\text { Leaf } \\
\text { stalks are } \\
\text { eaten raw } \\
\text { and used } \\
\text { as pickle. } \\
\text { Roots are } \\
\text { used as } \\
\text { substitute } \\
\text { of tea. } \\
\text { Also used } \\
\text { for } \\
\text { colouring } \\
\text { wool and } \\
\text { as } \\
\text { fixative }\end{array}$ \\
\hline 63 & $\begin{array}{l}\text { Rheum } \\
\text { moorcroftian } \\
\text { um }\end{array}$ & Polygonaceae & & Padamchal & $\begin{array}{l}\text { Himalayan } \\
\text { rhubarb }\end{array}$ & Root & $\begin{array}{l}\text { Fever, gastritis } \\
\text { and swellings }\end{array}$ & $\begin{array}{l}\text { Leaf } \\
\text { stalks are } \\
\text { eaten raw } \\
\text { and used } \\
\text { as pickle. } \\
\text { Roots are } \\
\text { used as } \\
\text { substitute } \\
\text { of tea }\end{array}$ \\
\hline 64 & $\begin{array}{l}\text { Rumex } \\
\text { nepalensis }\end{array}$ & Polygonaceae & Shyomang & $\begin{array}{l}\text { Ban } \\
\text { Palungo/H } \\
\text { alhale }\end{array}$ & $\begin{array}{l}\text { Sorrel/Yello } \\
\text { w doek }\end{array}$ & $\begin{array}{l}\text { Whole } \\
\text { plant }\end{array}$ & $\begin{array}{l}\text { Fever, cough, } \\
\text { blood clotting, } \\
\text { excretory } \\
\text { system and oral } \\
\text { disorders, joint } \\
\text { pain, wounds, }\end{array}$ & $\begin{array}{l}\text { Leaf } \\
\text { stalks are } \\
\text { eaten raw } \\
\text { and used } \\
\text { as pickle }\end{array}$ \\
\hline
\end{tabular}


M.R. Pandey / Our Nature (2006)4:69-82

\begin{tabular}{|c|c|c|c|c|c|c|c|c|}
\hline & & & & & & & $\begin{array}{l}\text { lung and liver } \\
\text { diseases, } \\
\text { constipation, } \\
\text { sores and skin } \\
\text { diseases }\end{array}$ & \\
\hline 65 & $\begin{array}{l}\text { Rumex } \\
\text { patientia }\end{array}$ & Polygonaceae & Shyomang & $\begin{array}{l}\text { Ban } \\
\text { Palungo/H } \\
\text { alhale }\end{array}$ & $\begin{array}{l}\text { Sorrel/Yello } \\
\text { w doek }\end{array}$ & Root & $\begin{array}{l}\text { Swellings and } \\
\text { constipation }\end{array}$ & $\begin{array}{l}\text { Leaf } \\
\text { stalks are } \\
\text { eaten raw } \\
\text { and used } \\
\text { as pickle }\end{array}$ \\
\hline 66 & $\begin{array}{l}\text { Androsace } \\
\text { muscoidea }\end{array}$ & Primulaceae & Pangatum & & $\begin{array}{l}\text { Rock } \\
\text { Jasmine }\end{array}$ & Flower & $\begin{array}{l}\text { Fever and } \\
\text { urinary } \\
\text { problems }\end{array}$ & \\
\hline 67 & $\begin{array}{l}\text { Androsace } \\
\text { sp. }\end{array}$ & Primulaceae & Pangatum & & $\begin{array}{l}\text { Rock } \\
\text { Jasmine }\end{array}$ & $\begin{array}{l}\text { Leaf } \\
\text { and } \\
\text { flower }\end{array}$ & $\begin{array}{l}\text { Cough, lung } \\
\text { and throat } \\
\text { problems }\end{array}$ & \\
\hline 68 & $\begin{array}{l}\text { Androsace } \\
\text { strigillosa }\end{array}$ & Primulaceae & $\begin{array}{l}\text { Gatik- } \\
\text { chungwa }\end{array}$ & & $\begin{array}{l}\text { Rock } \\
\text { Jasmine }\end{array}$ & $\begin{array}{l}\text { Flower } \\
\text { and } \\
\text { leaf }\end{array}$ & $\begin{array}{l}\text { Fever, body } \\
\text { swelling and } \\
\text { lymph disorder }\end{array}$ & \\
\hline 69 & $\begin{array}{l}\text { Primula } \\
\text { macrophylla }\end{array}$ & Primulaceae & $\begin{array}{l}\text { Shang } \\
\text { Dril } \\
\text { Nagpo }\end{array}$ & Primula & $\begin{array}{l}\text { Prime } \\
\text { Rose/Primu } \\
\text { la }\end{array}$ & $\begin{array}{l}\text { Whole } \\
\text { plant }\end{array}$ & $\begin{array}{l}\text { Food } \\
\text { poisoning, } \\
\text { fever, } \\
\text { indigestion, } \\
\text { dysentery and } \\
\text { ulcer }\end{array}$ & \\
\hline 70 & $\begin{array}{l}\text { Primula } \\
\text { sikkimensis }\end{array}$ & Primulaceae & & Primula & $\begin{array}{l}\text { Prime } \\
\text { Rose/Primu } \\
\text { la }\end{array}$ & Flower & $\begin{array}{l}\text { Fevers of lung, } \\
\text { blood vein } \\
\text { disorders and } \\
\text { diarrhoea }\end{array}$ & $\begin{array}{l}\text { Generally } \\
\text { used in } \\
\text { case of } \\
\text { children }\end{array}$ \\
\hline 71 & $\begin{array}{l}\text { Aconitum } \\
\text { naviculare }\end{array}$ & Ranunculaceae & Pogmar & Bish & $\begin{array}{l}\text { Aconite/Ati } \\
\text { s root }\end{array}$ & Tuber & $\begin{array}{l}\text { Fever, gall } \\
\text { bladder } \\
\text { disorder, food } \\
\text { poisoning and } \\
\text { communicable } \\
\text { diseases }\end{array}$ & \\
\hline 72 & $\begin{array}{l}\text { Anemone } \\
\text { rivularis }\end{array}$ & Ranunculaceae & $\begin{array}{l}\text { Supka } \\
\text { Karpo }\end{array}$ & Pati Dhuk & $\begin{array}{l}\text { Pasque } \\
\text { flower/Ane } \\
\text { mone }\end{array}$ & $\begin{array}{l}\text { Fruit } \\
\text { and } \\
\text { seed }\end{array}$ & $\begin{array}{l}\text { Wound, } \\
\text { accumulation } \\
\text { of serous fluid } \\
\text { in the body, } \\
\text { gastritis, liver } \\
\text { and bile } \\
\text { disorders, } \\
\text { cough, cold and } \\
\text { for heat } \\
\text { production }\end{array}$ & \\
\hline 73 & $\begin{array}{l}\text { Anemone } \\
\text { rupicola }\end{array}$ & Ranunculaceae & $\begin{array}{l}\text { Supka } \\
\text { Karpo }\end{array}$ & Pati Dhuk & $\begin{array}{l}\text { Pasque } \\
\text { flower/Ane } \\
\text { mone }\end{array}$ & Seed & Indigestion & \\
\hline 74 & $\begin{array}{l}\text { Clematis } \\
\text { tibetana }\end{array}$ & Ranunculaceae & Ngihmong & $\begin{array}{l}\text { Junge } \\
\text { Lahara }\end{array}$ & $\begin{array}{l}\text { Old man } \\
\text { beard }\end{array}$ & $\begin{array}{l}\text { Root, } \\
\text { stem, } \\
\text { leaf, } \\
\text { flower } \\
\text { and } \\
\text { fruit }\end{array}$ & $\begin{array}{l}\text { Removal of } \\
\text { accumulated } \\
\text { fluid from the } \\
\text { body, cold } \\
\text { tumours, } \\
\text { cough, cold, } \\
\text { indigestion, } \\
\text { joint pain, } \\
\text { swellings, } \\
\text { constipation, }\end{array}$ & \\
\hline
\end{tabular}


M.R. Pandey / Our Nature (2006)4:69-82

\begin{tabular}{|c|c|c|c|c|c|c|c|c|}
\hline & & & & & & & $\begin{array}{l}\text { wound } \\
\text { infection, as } \\
\text { appetizer and } \\
\text { for heat } \\
\text { production }\end{array}$ & \\
\hline 75 & $\begin{array}{l}\text { Delphinium } \\
\text { sp. }\end{array}$ & Ranunculaceae & Jakang & Nirmasi & $\begin{array}{l}\text { Aconite/Lar } \\
\text { kspur }\end{array}$ & $\begin{array}{l}\text { Leaf } \\
\text { and } \\
\text { flower }\end{array}$ & $\begin{array}{l}\text { Diarrhoea, } \\
\text { intestinal } \\
\text { disorders, } \\
\text { wounds and } \\
\text { used to kill lice }\end{array}$ & \\
\hline 76 & $\begin{array}{l}\text { Thalictrum } \\
\text { sp. }\end{array}$ & Ranunculaceae & Chag Kyu & $\begin{array}{l}\text { Bansuli/Da } \\
\text { mpate }\end{array}$ & $\begin{array}{l}\text { Meadow } \\
\text { rue }\end{array}$ & $\begin{array}{l}\text { Root, } \\
\text { leaf } \\
\text { and } \\
\text { flower }\end{array}$ & $\begin{array}{l}\text { Wounds and } \\
\text { infection, fever, } \\
\text { antiseptic and } \\
\text { poisoning }\end{array}$ & \\
\hline 77 & $\begin{array}{l}\text { Cotoneaster } \\
\text { sp. }\end{array}$ & Rosaceae & Chadung & $\begin{array}{l}\text { Dhalke } \\
\text { Phool }\end{array}$ & Cotoneaster & $\begin{array}{l}\text { Whole } \\
\text { plant }\end{array}$ & $\begin{array}{l}\text { Menstrual } \\
\text { disorder and } \\
\text { joint pain }\end{array}$ & \\
\hline 78 & $\begin{array}{l}\text { Potentilla } \\
\text { fruticosa }\end{array}$ & Rosaceae & $\begin{array}{l}\text { Pemma } \\
\text { Nhakpo }\end{array}$ & $\begin{array}{l}\text { Bhairab } \\
\text { Pati }\end{array}$ & $\begin{array}{l}\text { Shrubby } \\
\text { cinquefoil/ } \\
\text { Bush } \\
\text { cinquefoil }\end{array}$ & $\begin{array}{l}\text { Stem, } \\
\text { leaf } \\
\text { and } \\
\text { flower }\end{array}$ & $\begin{array}{l}\text { Breast diseases, } \\
\text { stomach and } \\
\text { lung disorders, } \\
\text { indigestion and } \\
\text { dysentery }\end{array}$ & $\begin{array}{l}\text { Plants are } \\
\text { also used } \\
\text { as } \\
\text { incense. } \\
\text { Leaves } \\
\text { are used } \\
\text { as a } \\
\text { substitute } \\
\text { for tea }\end{array}$ \\
\hline 79 & $\begin{array}{l}\text { Rosa } \\
\text { macrophylla }\end{array}$ & Rosaceae & Segoe Fo & $\begin{array}{l}\text { Jungali } \\
\text { Gulab }\end{array}$ & Wild Rose & Fruit & $\begin{array}{l}\text { Fever, } \\
\text { diarrhoea and } \\
\text { bile disorders }\end{array}$ & $\begin{array}{l}\text { Fruits can } \\
\text { be taken } \\
\text { raw and } \\
\text { also used } \\
\text { for } \\
\text { making } \\
\text { wine. }\end{array}$ \\
\hline 80 & Rosa sericea & Rosaceae & Se Gyoh & $\begin{array}{l}\text { Bhote } \\
\text { Gulab }\end{array}$ & $\begin{array}{l}\text { Wild } \\
\text { Rose/Himal } \\
\text { ayan Rose }\end{array}$ & $\begin{array}{l}\text { Flower } \\
\text {, fruit } \\
\text { and } \\
\text { bark }\end{array}$ & $\begin{array}{l}\text { Fever, } \\
\text { jaundice, liver, } \\
\text { bile, wind and } \\
\text { lung diseases, } \\
\text { menstrual } \\
\text { disorders, } \\
\text { poisoning, } \\
\text { lymph fluid } \\
\text { disorder and as } \\
\text { tonic }\end{array}$ & $\begin{array}{l}\text { Ripe } \\
\text { fruits can } \\
\text { be taken } \\
\text { raw. } \\
\text { Flowers } \\
\text { are used } \\
\text { as facial } \\
\text { wash. } \\
\text { This plant } \\
\text { is used as } \\
\text { herbal } \\
\text { tea. }\end{array}$ \\
\hline 81 & Salix sp. & Salicaceae & & Bains & Willow & Stem & $\begin{array}{l}\text { Venereal } \\
\text { disease, uterus } \\
\text { problem, fever } \\
\text { and arthritis } \\
\end{array}$ & \\
\hline 82 & $\begin{array}{l}\text { Lagotis } \\
\text { kunawurensi } \\
\text { s }\end{array}$ & Scrophulariaceae & Hoanglen & Baraka & Lagotis & $\begin{array}{l}\text { Whole } \\
\text { plant }\end{array}$ & $\begin{array}{l}\text { Diarrhoea, T. } \\
\text { B., Pyrexia } \\
\text { unknown origin } \\
\text { (PUO) and } \\
\text { excess bleeding } \\
\text { from the } \\
\text { wound }\end{array}$ & \\
\hline
\end{tabular}


M.R. Pandey / Our Nature (2006)4:69-82

\begin{tabular}{|c|c|c|c|c|c|c|c|c|}
\hline 83 & $\begin{array}{l}\text { Lancea } \\
\text { tibetica }\end{array}$ & Scrophulariaceae & $\begin{array}{l}\text { Payokcha } \\
\text { wa }\end{array}$ & & & $\begin{array}{l}\text { Root, } \\
\text { leaf, } \\
\text { flower } \\
\text { and } \\
\text { berry }\end{array}$ & $\begin{array}{l}\text { Lung and } \\
\text { urinary bladder } \\
\text { problems } \\
\text { mainly urine } \\
\text { blocked, } \\
\text { wound } \\
\text { infection, fever, } \\
\text { cuts, wounds, } \\
\text { menstruation } \\
\text { problem, heart } \\
\text { disease and } \\
\text { problems of } \\
\text { veins }\end{array}$ & \\
\hline 84 & $\begin{array}{l}\text { Pedicularis } \\
\text { longiflora }\end{array}$ & Scrophulariaceae & $\begin{array}{l}\text { Lukru } \\
\text { Serpo }\end{array}$ & Jumre Jhar & Lousewort & $\begin{array}{l}\text { Flower } \\
\text { and } \\
\text { whole } \\
\text { plant }\end{array}$ & $\begin{array}{l}\text { Food } \\
\text { poisoning, } \\
\text { stomach and } \\
\text { lymph fluid } \\
\text { disorders, T. } \\
\text { B., diarrhoea, } \\
\text { asthma, wound } \\
\text { infection, } \\
\text { premature } \\
\text { ejaculation, } \\
\text { fever due to } \\
\text { disorders of } \\
\text { liver and gall } \\
\text { bladder }\end{array}$ & $\begin{array}{l}\text { Also used } \\
\text { as hair } \\
\text { dye }\end{array}$ \\
\hline 85 & $\begin{array}{l}\text { Verbascum } \\
\text { thapsus }\end{array}$ & Scrophulariaceae & Nho Sarje & $\begin{array}{l}\text { Gan } \\
\text { Puchchhre }\end{array}$ & Mullein & $\begin{array}{l}\text { Whole } \\
\text { plant } \\
\text { and } \\
\text { root }\end{array}$ & $\begin{array}{l}\text { Swellings, } \\
\text { asthma, cough, } \\
\text { blood disorder, } \\
\text { bleeding, } \\
\text { wounds and } \\
\text { cuts }\end{array}$ & \\
\hline 86 & $\begin{array}{l}\text { Hyoscyamus } \\
\text { niger }\end{array}$ & Solanaceae & $\begin{array}{l}\text { Langh } \\
\text { Thang } \\
\text { Che }\end{array}$ & $\begin{array}{l}\text { Bajra } \\
\text { Bhang }\end{array}$ & Henbane & Seed & $\begin{array}{l}\text { Sinusitis, } \\
\text { intestinal } \\
\text { parasites, } \\
\text { diarrhoea and } \\
\text { worm } \\
\text { infections } \\
\end{array}$ & \\
\hline 87 & $\begin{array}{l}\text { Stellera } \\
\text { chamaejasme }\end{array}$ & Thymelaeaceae & Ri Jyakpa & $\begin{array}{l}\text { Ramro } \\
\text { Phool/Reke } \\
\text { mukta }\end{array}$ & Stellera & Root & $\begin{array}{l}\text { Poultice for } \\
\text { swellings and } \\
\text { fractured } \\
\text { bones, for } \\
\text { massage, } \\
\text { antiseptic for } \\
\text { cuts and } \\
\text { wounds, } \\
\text { analgesic, } \\
\text { infectious } \\
\text { diseases, } \\
\text { gastritis and } \\
\text { ulcer }\end{array}$ & $\begin{array}{l}\text { Smell of } \\
\text { the flower } \\
\text { can cause } \\
\text { headache. }\end{array}$ \\
\hline
\end{tabular}


M.R. Pandey / Our Nature (2006)4:69-82

\begin{tabular}{|c|c|c|c|c|c|c|c|c|}
\hline 88 & Carum carvi & Umbelliferae & Gyoh Nge & Bhote Jira & $\begin{array}{l}\text { Himalayan } \\
\text { Caraway }\end{array}$ & $\begin{array}{l}\text { Whole } \\
\text { plant }\end{array}$ & $\begin{array}{l}\text { Cold, cough, } \\
\text { fever, food } \\
\text { poisoning, eye } \\
\text { infection, } \\
\text { gastric } \\
\text { dysfunction, } \\
\text { stomachache } \\
\text { and } \\
\text { carminative }\end{array}$ & $\begin{array}{l}\text { The seeds } \\
\text { are used } \\
\text { for the } \\
\text { preparatio } \\
\mathrm{n} \text { of } \\
\text { massage } \\
\text { oil for } \\
\text { baby. The } \\
\text { powder of } \\
\text { the seed } \\
\text { is } \\
\text { employed } \\
\text { to flavour } \\
\text { vegetable } \\
\text { s and } \\
\text { curries }\end{array}$ \\
\hline 89 & $\begin{array}{l}\text { Cortia } \\
\text { depressa }\end{array}$ & Umbelliferae & $\begin{array}{l}\text { Tang } \\
\text { Kyun }\end{array}$ & & & $\begin{array}{l}\text { Flower } \\
\text { and } \\
\text { leaf }\end{array}$ & $\begin{array}{l}\text { Fever, } \\
\text { rheumatism, } \\
\text { sedative and } \\
\text { stomachache }\end{array}$ & \\
\hline 90 & $\begin{array}{l}\text { Heracleum } \\
\text { lallii }\end{array}$ & Umbelliferae & Tukar & $\begin{array}{l}\text { Bhoot } \\
\text { Kesh }\end{array}$ & & $\begin{array}{l}\text { Root } \\
\text { and } \\
\text { fruit }\end{array}$ & $\begin{array}{l}\text { Stomach } \\
\text { disorders, } \\
\text { earache, } \\
\text { leprosy, joint } \\
\text { pain and blood } \\
\text { pressure }\end{array}$ & \\
\hline 91 & $\begin{array}{l}\text { Selinum } \\
\text { tenuifolium }\end{array}$ & Umbelliferae & Tunak & $\begin{array}{l}\text { Jwane } \\
\text { Ghans/Bha } \\
\text { draji }\end{array}$ & Ragwort & Root & $\begin{array}{l}\text { Antiseptic, } \\
\text { gastritis, } \\
\text { hemorrhage, } \\
\text { cough and cold }\end{array}$ & \\
\hline 92 & $\begin{array}{l}\text { Nardostachy } \\
\text { s grandiflora }\end{array}$ & Valerianaceae & Pangpyo & Jatamasi & $\begin{array}{l}\text { Spikenard/ } \\
\text { Valerian }\end{array}$ & $\begin{array}{l}\text { Whole } \\
\text { plant }\end{array}$ & $\begin{array}{l}\text { Lung, heart and } \\
\text { liver problems, } \\
\text { wounds, cough, } \\
\text { cold, chronic } \\
\text { fever, fever due } \\
\text { to poisoning, } \\
\text { spleen disease, } \\
\text { intestinal } \\
\text { parasites, } \\
\text { hypertension, } \\
\text { tumours, } \\
\text { swellings, high } \\
\text { altitude } \\
\text { sickness, } \\
\text { headache, } \\
\text { epilepsy, } \\
\text { hysteria, skin } \\
\text { diseases, throat } \\
\text { trouble, } \\
\text { lumbago, } \\
\text { ulcers, } \\
\text { rheumatism, } \\
\text { paralysis and } \\
\text { diuretic }\end{array}$ & $\begin{array}{l}\text { Whole } \\
\text { plant is } \\
\text { used as } \\
\text { incense } \\
\text { with } \\
\text { Rhododen } \\
\text { dron } \\
\text { anthopog } \\
\text { on, } \\
\text { Tanacetu } \\
m \\
\text { nubigenu } \\
\text { m and } \\
\text { Juniperus } \\
\text { spp. }\end{array}$ \\
\hline 93 & Viola biflora & Violaceae & Ta Mig & $\begin{array}{l}\text { Panhelo } \\
\text { Ghatte } \\
\text { Phool }\end{array}$ & $\begin{array}{l}\text { Violet/Wild } \\
\text { pancy }\end{array}$ & $\begin{array}{l}\text { Whole } \\
\text { plant }\end{array}$ & $\begin{array}{l}\text { Wounds, } \\
\text { fractured } \\
\text { bones, bile and } \\
\text { head disorders }\end{array}$ & \\
\hline
\end{tabular}

